\section{Análisis preliminares de la versión adaptada en población chilena de la escala de adherencia terapéutica en diabetes mellitus tipo 2 - EATDM-III}

\author{
ALFONSO URZÚA M., ,a, CARLOS CABRERA R. ${ }^{1, \mathrm{~b}}$, \\ CHRISTOFER GONZÁLEZ V..$^{1, b}$, PABLO ARENAS R..$^{1, b}$, \\ MÓNICA GUZMÁN G. ${ }^{1, \mathrm{c}}$, ALEJANDRA CAQUEO-URÍZAR ${ }^{2, \mathrm{~d}}$, \\ ALFONSO VILLALOBOS P. ${ }^{3, \mathrm{e}}$, MATÍAS IRARRÁZAVAL D. ${ }^{4,5}$
}

\section{Psychometric properties of the diabetes mellitus 2 treatment adherence scale version III (EATDM-III) adapted for Chilean patients}

Background: The Diabetes Mellitus 2 treatment adherence scale version III (EATDM-III) was devised in Costa Rica. Its seven factors are family support, community organization and support, physical exercise, medical control, hygiene and self-care and assessment of physical condition. Aim: To assess the psychometric properties of the scale in Chilean patients. Material and Methods: The results of the EATDM-III scale, applied to 274 patients with Diabetes Mellitus 2 aged $59 \pm 11$ years (59\% women), were analyzed. Reliability, item, exploratory and confirmatory factorial analyses were carried out both in the initial and the proposed model. Results: We propose a version of 30 items grouped in six dimensions, improving the fit indices obtained with the original scale. The review of item factor loadings shows that all are appropriate both in magnitude and statistical significance, with values between 0.46 and 0.93 . Internal consistency measured by Cronbach's alpha, was 0.85 for the total scale. Conclusions: The adapted EATDM-III scale is reliable and can be used to assess treatment adherence in Chilean patients.

(Rev Med Chile 2015; 143: 733-743)

Key words: Diabetes Mellitus; Patient compliance; Psychometrics.
'Escuela de Psicología,

Universidad Católica del Norte, Antofagasta, Chile.

${ }^{2}$ Departamento de Filosofía y Psicología, Facultad de Ciencias Sociales y Jurídicas, Universidad de Tarapacá, Arica, Chile.

${ }^{3}$ Caja Costarricense de Seguro Social, San José Costa Rica.

${ }^{4}$ Departamento de Psiquiatría

Norte, Universidad de Chile,

Hospital Clínico Universidad de

Chile. Santiago de Chile.

${ }^{5}$ Departamento de Psiquiatría

Infantil y Adolescente, Clínica

Las Condes.

apsicólogo, Magíster en Salud

Pública, Doctor en Psicología

Clínica y de la Salud.

bPsicólogo, Licenciado en

Psicología.

'Psicóloga, Doctora en Psicología.

dPsicóloga, Magíster en

Psicopatología Infanto-Juvenil,

Doctora en Psicología Clínica y de

la Salud.

ePsicólogo, Magíster en Ciencias Cognitivas, Universidad Estatal a

Distancia, Costa Rica.

Recibido el 11 de marzo de 2013, aceptado el 26 de abril de 2015.

Correspondencia a:

Dr. Alfonso Urzúa

Avenida Angamos 0610,

Antofagasta, Chile.

alurzua@ucn.cl
E n Chile, la prevalencia general de diabetes mellitus (DM) en el 2009 fue de 9,4\%, elevándose a $16,9 \%$ en el grupo de 45-64 años y a $25,8 \%$ en el de 65 y más años. La DM tipo 2 (DM2) constituye $80-90 \%$ de los casos diagnosticados ${ }^{1}$.

El alto número de personas con DM implica no tan sólo enormes gastos para la salud pública, sino también costos considerables para la persona que vive con la enfermedad. La DM2 afecta negativamente la calidad de vida relacionada con la salud (CVRS), diversos factores se han reportado vinculados a una mejor o peor CVRS en personas con DM2, tales como el sexo ${ }^{2-4}$, edad ${ }^{2,5,6}$, la presencia de la pareja ${ }^{4}$, nivel de estudios ${ }^{3,5,7}$, apoyo social $^{8}$, índice de masa corporal ${ }^{2,9}$, complicaciones derivadas ${ }^{2,5,7,10}$, comorbilidades asociadas ${ }^{9}$, tipo de tratamiento y satisfacción con este ${ }^{3}$, control glucémico ${ }^{3,9} \mathrm{y}$ satisfacción con la salud ${ }^{6,11}$.

Esta patología se complica debido a la falta de adherencia a su tratamiento, definida como el grado en que la conducta de una persona (tomar 
el medicamento, seguir un régimen alimentario y ejecutar cambios del modo de vida), se corresponde con las recomendaciones acordadas por un prestador de asistencia sanitaria ${ }^{12}$.

En países desarrollados sólo entre 45\% y 50\% de los enfermos crónicos cumple con el tratamiento, siendo menor en países en desarrollo ${ }^{13}$. Esta situación se agrava en los países pobres, donde el acceso a los medicamentos está limitado por la falta de recursos ${ }^{12}$. Las bajas tasas de adherencia apoyan la idea de que el paciente mayoritariamente no cumple con el tratamiento ${ }^{14}$, en especial cuando los regímenes consisten en cambios de hábitos o estilos de vida ${ }^{15,16}$.

La baja adherencia afecta la calidad de vida del enfermo y de quienes están a su alrededor. En lo personal, el paciente puede tener complicaciones y secuelas que traen consigo un gran sufrimiento, así como limitaciones incontrolables y progresivas. En la familia, provoca alteraciones en su funcionamiento, generando crisis. En lo social, significa un enorme costo para las instituciones de salud el proporcionar servicios que son utilizados de forma inadecuada, prolongando innecesariamente los tratamientos y presentando recaídas y readmisiones que podrían evitarse ${ }^{17}$.

Entre los factores que dificultan la adherencia al tratamiento, se encuentran: concepciones culturales de la enfermedad, desencuentro entre los discursos del equipo profesional y el saber popular, cansancio de ingerir una cantidad elevada de medicamentos, temor a las múltiples punciones por la aplicación de la insulina, insatisfacción con la calidad brindada de los servicios de salud, el costo económico e incluso el estrés ${ }^{18,19}$.

La medición de la conducta de adherencia es necesaria para la planificación de tratamientos efectivos y eficientes, sin embargo, en el caso de la DM esto ha sido un problema, no sólo por el número de conductas que han de evaluarse, sino también por las dimensiones indicadoras de adherencia en cada una de ellas, así como entre las conductas a evaluar ${ }^{20}$.

No existe un "patrón de oro" para medir la conducta de adherencia terapéutica, reportándose una variedad de estrategias ${ }^{12}$. Un enfoque ha sido solicitar reportes subjetivos a prestadores y pacientes, sin embargo, los prestadores sobreestiman el grado en que los pacientes siguen sus recomendaciones, en tanto que los pacientes que revelan no haber seguido las recomendaciones terapéuticas tienden a describir su comportamiento con exactitud, mientras que los que niegan el no haberlas seguido informan sobre su conducta inexactamente ${ }^{12}$.

En este contexto, una propuesta destinada a evaluar diversos factores conductuales vinculados a la adherencia terapéutica en pacientes con DM2 es la Escala EATDM-III. Dado que la DM2, aunque es una enfermedad con implicaciones específicas en el deterioro físico de las personas que la padecen, implica una serie de elementos de tipo conductual, cognitivo y emocional que parecen estar condicionados por elementos culturales, propios de las estrategias sociales, y algunos estilos de atención médica de una zona geográfica, es que se hace necesario evaluar la adaptación de este instrumento a otras realidades, considerando factores ambientales como la cultura o las situaciones sociales propias de los sujetos bajo estudio ${ }^{20}$.

Dados los buenos resultados que ha tenido su aplicación inicial y además por la viabilidad de aplicarla en contextos de atención primaria en salud (APS), es que el objetivo de esta investigación fue la adaptación y evaluación de las propiedades psicométricas de la escala de adherencia al tratamiento de la DM2-Versión III (EATDM-III).

\section{Pacientes y Método}

\section{Participantes}

El proceso constó de tres etapas. En una primera fase, se aplicó la escala a 12 personas con DM2, 8 mujeres y cuatro hombres, usuarias de APS, con una media de edad de 62,35 años (DE = 5,32 ). La segunda etapa consideró la aplicación de la escala a una población de 50 personas con DM2, usuarias de APS. De éstas, 36 fueron mujeres $(72 \%)$ y 14 hombres (28\%). El promedio de edad en esta fase fue de 63,92 años (DE $=10,02)$. Los participantes en la tercera fase correspondieron a 274 personas diagnosticadas con DM2, con edades entre los 23 y los 93 años ( $\overline{\mathrm{x}}=58,71$; DE $=11,37)$, de éstos, 161 eran mujeres $(58,8 \%)$ con un promedio de edad de 58,55 años $(\mathrm{DE}=12,06)$ y 113 hombres $(41,2 \%)$ con una media de 58,95 años (DE $=10,37)$. Todos ellos beneficiarios del Fondo Nacional de Salud (FONASA), usuarios de APS $(90 \%)$ o de consultas privadas bajo modalidad FONASA. 


\section{Instrumento}

La escala de adherencia al tratamiento de la DM2-Versión III (EATDM-III) fue creada en Costa Rica. Está conformada por 55 ítems distribuidos en 7 factores: apoyo familiar, organización y apoyo comunal, ejercicio físico, control médico, higiene y autocuidado, dieta y valoración de la condición física. La escala de auto aplicación ofrece 5 tipos de respuesta tipo Likert que oscilan desde la ausencia de la conducta, valorada en 0 (nunca), hasta 4 puntos (siempre). Se ha reportado una consistencia interna con alfa de Cronbach que oscilan entre 0,70 y 0,84 para los diversos factores $\mathrm{y}$ de un 0,88 para la escala completa ${ }^{20} \mathrm{y}$ un análisis de componentes principales en que los factores explicaban $50 \%$ de la varianza total ${ }^{21}$.

\section{Procedimientos}

Una vez obtenidos los permisos necesarios de los autores de la escala para realizar su adaptación en Chile, y de contar con la evaluación del Comité de Bioética de la Universidad Católica del Norte, el procedimiento para la adaptación se realizó en tres fases.

Primero se evaluó la comprensión de los ítems que componían la escala. Los cuestionarios fueron recogidos entre mayo y septiembre de 2009 por tres internos de psicología capacitados en procedimientos de recolección de información. El tiempo promedio de llenado de la escala fue entre 10 y $15 \mathrm{~min}$. Cada vez que un cuestionario era completado, se realizó una entrevista cognitiva a la persona evaluada, a fin de recoger información acerca de cuáles eran las preguntas con mayor nivel de dificultad, o bien que no habían sido comprendidas, a fin de asegurar la equivalencia conceptual y semántica de la escala. Luego se procedió a corregir y adaptar las preguntas originales que no habían sido comprendidas por los participantes. Considerando que en las entrevistas surgieron elementos vinculados a la adherencia que no estaban contemplados en el cuestionario original, se adicionaron 10 reactivos. Con esto la escala quedó compuesta por 65 reactivos para ser aplicada en la etapa posterior.

Con los datos obtenidos en la segunda fase $(\mathrm{n}=50)$, se calculó la correlación de cada reactivo con la escala total, eliminándose los ítems cuyo valor estaba por debajo de 0,3 .

La escala final, constituida por 41 reactivos, fue aplicada a 274 usuarios del programa de control de crónicos de la APS y del Hospital Clínico Regional de Antofagasta y pacientes de consultas particulares, todos estos beneficiarios del Fondo Nacional de Salud, esto correspondió a la tercera fase.

Todos los participantes firmaron un consentimiento informado, el cual, en el caso del hospital y APS, permitió el acceso a los datos de su ficha clínica. La evaluación se realizó de manera individual en las salas de espera de los establecimientos. En el caso de los pacientes con déficit visual, las encuestas fueron leídas en forma individual.

Tanto el estadístico de adecuación muestral KMO $(0,78)$ como la prueba de esfericidad de Bartlett $\left(\chi_{(820)}^{2}=4.855,84 ; \mathrm{p}<0,01\right)$, indicaron la pertinencia de la realización de un análisis factorial. Dada la relación teórica entre las dimensiones se decide utilizar el método de extracción de ejes principales, con rotación VARIMAX.

Considerando el gráfico de saturación, se opta por una solución de seis factores como la más apropiada: ejercicio físico (EF), apoyo familiar (AF), control y tratamiento médico (CT), apoyo y organización comunal (AOC), dieta (D) e información (I). Estos factores explican 49,45\% de la varianza. Se procede a analizar la pertinencia y saturación de los ítems en los diversos factores, eliminándose 01 reactivo (consumo ocho vasos de agua diarios), ya que saturó indistintamente en EF y DIE.

Los 40 reactivos restantes fueron sometidos al mismo procedimiento de análisis, el cual fue pertinente dado el estadístico de adecuación muestral KMO $(0,78)$ como la prueba de esfericidad de Bartlett $\left(\chi_{(780)}^{2}=4.767,49 ; \mathrm{p}<0,01\right)$, indicaron la pertinencia de la realización de un análisis factorial. Los seis factores fueron capaces de explicar 50,52\% de la varianza. El análisis de los reactivos brinda la posibilidad de eliminar cuatro reactivos, los cuales a pesar de la congruencia teórica con el factor correspondiente, presentaban una saturación muy baja en este (menor a 0,4 ).

Con esta nueva solución, se procede a realizar un nuevo análisis factorial con los 36 reactivos restantes, así como a calcular la consistencia interna de la escala total y de cada uno de los factores, análisis de ítems (correlación ítem test, correlación ítem factor, asimetría, curtosis). Considerando la distribución desigual del número de preguntas por factor y a fin de facilitar la 
comprensión de los resultados para su utilización en la práctica clínica, la sintaxis de corrección incorporó la conversión de los puntajes de cada dominio a una escala de 0 a 100 puntos, donde el mayor puntaje expresa una mayor adherencia en ese factor.

\section{Resultados}

\section{Descripción de los participantes}

La muestra de la etapa final se conformó por 274 sujetos, de los cuales 161 eran mujeres $(58,8 \%)$ y 113 hombres $(41,2 \%)$. La mayor parte de los sujetos tenía un nivel socioeconómico bajo $(42,7 \%$ posee un ingreso familiar menor de \$127.668). Las características de esta población en términos socio-demográficos y estado de salud pueden ser vistas en la Tabla 1.

\section{Estructura factorial}

Tanto el estadístico de adecuación muestral $\mathrm{KMO}(0,78)$ como la prueba de esfericidad de Bartlett $\left(\chi_{(630)}^{2}=4.426,55 ; \mathrm{p}<0,01\right)$, indicaron la pertinencia de la realización de un análisis factorial. Los seis factores explican 54,04\% de la varianza. Ejercicio físico 11,21\%; apoyo familiar $11,14 \%$; control y tratamiento médico 8,46\%; apo- yo y organización comunal 8,07\%; dieta 5,37\% e información $5,20 \%$. Todos ellos con un eigenvalue mayor que uno. La distribución de los reactivos y su carga factorial pueden ser observados en la matriz de componentes rotados (Tabla 2).

\section{Confiabilidad}

Se evaluó a través de la consistencia interna calculada a través del estadístico alfa de Cronbach. Se encontró alfas de 0,87 para la escala total, apoyo familiar $=0,84$; ejercicio físico $=0,88$; control y tratamiento médico $=0,79$; apoyo $\mathrm{y}$ organización comunal $=0,79$; información $=0,72$ $\mathrm{y}$ dieta $=0,57$.

\section{Análisis de items}

Las correlaciones ítem-total de la escala oscilaron entre 0,23 y 0,55 y las correlaciones ítem-dimensión entre 0,35 y 0,83 (Tabla 3 ).

La Tabla 4 contiene la distribución porcentual de las respuestas, así como la media obtenida en cada una de las preguntas, el valor mínimo y el valor máximo observado. Los valores estadísticos de asimetría tendieron a agruparse hacia la derecha de la curva, por encima de la media (valores negativos), así como también los valores de la curtosis, que tendieron a hacer la curva más plana.

Tabla 1. Características de los participantes

\begin{tabular}{|lccc|}
\hline & Hombres & Mujeres & Muestra Total \\
\hline Edad promedio (años) & $59 \pm 10,60$ & $58 \pm 12,05$ & $59 \pm 11,37$ \\
\hline Edad mínima/máxima & $25 / 82$ & $23 / 93$ & $23 / 93$ \\
\hline Ingreso familiar (Q1/Q5) & 3,2 & 7,6 & 5,6 \\
\hline Índice masa corporal & $28 \pm 3,70$ & $29 \pm 4,76$ & $28 \pm 4,41$ \\
\hline Peso (kilogramos) & $78 \pm 11,43$ & $71 \pm 11,66$ & $74 \pm 12,10$ \\
\hline Presión sistólica (mg/hg) & $134 \pm 20,24$ & $129 \pm 19,47$ & $131 \pm 19,88$ \\
\hline Presión diastólica (mg/hg) & $81 \pm 10,47$ & $77 \pm 11,44$ & $79 \pm 11,17$ \\
\hline Colesterol (mg/dl) & $205 \pm 42,19$ & $207 \pm 45,80$ & $206 \pm 44,32$ \\
\hline Glicemia & $169 \pm 65,90$ & $168 \pm 64,90$ & $169 \pm 65,11$ \\
\hline Con pareja & $96(85 \%)$ & $104(64,4 \%)$ & $200(73 \%)$ \\
\hline Con comorbilidad hipertensión & $82(72,6 \%)$ & $106(65,8 \%)$ & $188(68,6 \%)$ \\
\hline Con percepción de complicaciones derivadas de la DM & $20(17,7 \%)$ & $43(26,7 \%)$ & $63(23 \%)$ \\
\hline
\end{tabular}


Tabla 2. Matriz de componentes rotados

\begin{tabular}{|c|c|c|c|c|c|c|}
\hline & \multicolumn{6}{|c|}{ Componente } \\
\hline & $\mathbf{E F}$ & AF & CT & AOC & INF & DIE \\
\hline$\%$ varianza explicado & 12,82 & 12,41 & 8,98 & 8,78 & 6,07 & 4,98 \\
\hline Evito consumir golosinas o confites dulces & 0,157 & $-0,019$ & 0,227 & $-0,033$ & 0,290 & 0,600 \\
\hline Equilibro mi dieta consumiendo distintos tipos de alimentos & 0,302 & 0,234 & $-0,002$ & $-0,071$ & 0,093 & 0,598 \\
\hline Consumo frutas al menos una vez al día & 0,066 & 0,167 & $-0,004$ & 0,124 & 0,026 & 0,599 \\
\hline Camino para sentirme mejor & 0,612 & 0,057 & 0,055 & 0,103 & 0,048 & 0,198 \\
\hline Consumo alimentos antes de realizar ejercicio & 0,575 & $-0,086$ & $-0,118$ & 0,100 & 0,012 & 0,088 \\
\hline Tengo un horario fijo para realizar ejercicio & 0,684 & 0,114 & $-0,002$ & $-0,042$ & 0,075 & $-0,058$ \\
\hline Sufro de menos cansancio cuando realizo alguna actividad física & 0,777 & 0,029 & 0,011 & 0,114 & 0,033 & $-0,004$ \\
\hline Me siento menos triste o decaído cuando realizo una actividad física & 0,730 & $-0,019$ & 0,067 & 0,001 & 0,103 & $-0,054$ \\
\hline Mejoro mi condición física cuando realizo alguna actividad física & 0,871 & 0,058 & $-0,002$ & 0,106 & 0,056 & 0,054 \\
\hline Siento mejores resultados en el tratamiento después del ejercicio & 0,864 & 0,101 & 0,061 & 0,120 & 0,057 & $-0,004$ \\
\hline Realizo paseos cortos durante el día & 0,620 & $-0,022$ & 0,095 & 0,071 & $-0,148$ & 0,246 \\
\hline Visito al médico del consultorio en caso de alguna complicación & 0,170 & 0,160 & 0,556 & $-0,019$ & $-0,060$ & 0,160 \\
\hline Asisto puntualmente a las citas de control de diabetes & 0,008 & 0,095 & 0,819 & 0,020 & 0,016 & 0,031 \\
\hline Asisto al laboratorio para realizar las pruebas respectivas & 0,040 & 0,089 & 0,822 & 0,037 & $-0,088$ & 0,115 \\
\hline El equipo de salud me brinda información sobre mi enfermedad & 0,082 & 0,004 & 0,667 & 0,153 & 0,322 & $-0,132$ \\
\hline Estoy satisfecho con los resultados del tratamiento médico & $-0,031$ & 0,119 & 0,632 & 0,098 & 0,210 & 0,052 \\
\hline El tratamiento me permite controlar la enfermedad & $-0,119$ & 0,095 & 0,613 & 0,028 & 0,217 & $-0,035$ \\
\hline Mi familia permite que prepare mis alimentos aparte del de los demás & $-0,005$ & 0,468 & 0,227 & $-0,030$ & 0,147 & 0,239 \\
\hline Mis familiares me facilitan los alimentos que necesito para la dieta especial & 0,011 & 0,654 & 0,086 & 0,037 & 0,169 & 0,226 \\
\hline Mis familiares están pendientes de mis medicamentos durante el día & $-0,001$ & 0,760 & 0,097 & 0,130 & 0,021 & 0,135 \\
\hline $\begin{array}{l}\text { Mis familiares colaboran con los implementos de uso personal } \\
\text { especiales (zapatos, limas para uñas) }\end{array}$ & 0,070 & 0,674 & 0,164 & 0,177 & 0,113 & 0,034 \\
\hline $\begin{array}{l}\text { Recibo estímulos verbales (apoyo) de parte de mis familiares para } \\
\text { que no abandone el tratamiento }\end{array}$ & 0,049 & 0,716 & 0,078 & $-0,062$ & $-0,027$ & $-0,027$ \\
\hline $\begin{array}{l}\text { Recibo premios o recompensas de parte de mis familiares para que } \\
\text { no abandone el tratamiento }\end{array}$ & 0,126 & 0,526 & $-0,042$ & 0,189 & 0,189 & $-0,222$ \\
\hline Mis familiares me instan en la práctica de alguna actividad recreativa & 0,289 & 0,547 & $-0,057$ & $-0,027$ & 0,165 & $-0,319$ \\
\hline Mis familiares me instan a la práctica de algún deporte & 0,197 & 0,504 & $-0,079$ & 0,023 & 0,334 & $-0,379$ \\
\hline $\begin{array}{l}\text { Mis familiares están pendientes de que no pierda las citas de control } \\
\text { de la diabetes en el consultorio }\end{array}$ & $-0,052$ & 0,706 & 0,260 & 0,051 & $-0,183$ & 0,062 \\
\hline $\begin{array}{l}\text { Mis familiares están pendientes de que el baño u otros lugares de la } \\
\text { casa se encuentren limpios y desinfectados }\end{array}$ & $-0,134$ & 0,736 & $-0,022$ & 0,128 & $-0,094$ & 0,261 \\
\hline $\begin{array}{l}\text { Se realiza en la comunidad charlas educativas acerca de la diabetes } \\
\text { con el fin de informar a las personas }\end{array}$ & $-0,020$ & 0,078 & 0,073 & 0,689 & 0,254 & $-0,036$ \\
\hline $\begin{array}{l}\text { En la comunidad donde vivo se organizan en grupos para la práctica } \\
\text { de algún deporte }\end{array}$ & 0,053 & 0,100 & 0,041 & 0,648 & 0,030 & $-0,003$ \\
\hline En la comunidad donde vivo se realizan actividades recreativas & 0,030 & 0,043 & 0,079 & 0,715 & 0,004 & 0,066 \\
\hline $\begin{array}{l}\text { La comunidad se organiza para la realización de actividades como } \\
\text { la compra de medicamentos especiales para la diabetes }\end{array}$ & 0,142 & 0,034 & 0,025 & 0,752 & $-0,100$ & 0,053 \\
\hline $\begin{array}{l}\text { La comunidad se organiza para la realización de actividades como } \\
\text { la compra de instrumentos de control de glucemia }\end{array}$ & 0,165 & 0,023 & $-0,004$ & 0,723 & $-0,032$ & $-0,034$ \\
\hline Asisto a charlas brindadas por miembros del área de salud de mi comunidad & 0,101 & 0,108 & 0,043 & 0,626 & 0,273 & 0,029 \\
\hline Mi familia conoce lo que es la diabetes mellitus & $-0,008$ & 0,421 & 0,144 & $-0,007$ & 0,504 & 0,098 \\
\hline Alguna vez he recibido información sobre la diabetes mellitus & 0,108 & 0,091 & 0,154 & 0,172 & 0,778 & 0,106 \\
\hline Tengo claro en qué consiste la diabetes mellitus & 0,079 & 0,034 & 0,178 & 0,112 & 0,740 & 0,100 \\
\hline
\end{tabular}

Método de extracción: Análisis de componentes principales; Método de rotación: Normalización Varimax con Kaiser, La rotación ha convergido en 7 iteraciones, $\mathrm{EF}=$ Ejercicio físico; $\mathrm{AF}=$ Apoyo familiar; $\mathrm{CT}=$ Control y tratamiento médico; $\mathrm{AOC}=\mathrm{Apoyo}$ y organización comunal; DIE = Dieta; INF= Información. 


\section{Tabla 3. Correlaciones ítem-Total e Ítem-Dimensión, y alfa de Cronbach}

\begin{tabular}{|c|c|c|c|c|}
\hline & & CIT & CID & $\begin{array}{l}\text { Alfa de } \\
\text { Cronbach }\end{array}$ \\
\hline 1 & Evito consumir golosinas o confites dulces & 0,27 & 0,35 & \multirow{3}{*}{0,572} \\
\hline 2 & Equilibro mi dieta consumiendo distintos tipos de alimentos & 0,35 & 0,43 & \\
\hline 3 & Consumo frutas al menos una vez al día & 0,24 & 0,37 & \\
\hline 4 & Camino para sentirme mejor & 0,41 & 0,55 & \multirow{8}{*}{0,868} \\
\hline 5 & Consumo alimentos antes de realizar ejercicio & 0,23 & 0,47 & \\
\hline 6 & Tengo un horario fijo para realizar ejercicio & 0,38 & 0,58 & \\
\hline 7 & Sufro de menos cansancio cuando realizo alguna actividad física & 0,43 & 0,71 & \\
\hline 8 & Me siento menos triste o decaído cuando realizo una actividad física & 0,37 & 0,64 & \\
\hline 9 & Mejoro mi condición física cuando realizo alguna actividad física & 0,51 & 0,83 & \\
\hline 10 & Siento mejores resultados en el tratamiento después del ejercicio & 0,55 & 0,82 & \\
\hline 11 & Realizo paseos cortos durante el día & 0,32 & 0,52 & \\
\hline 12 & Visito al médico del consultorio en caso de alguna complicación & 0,32 & 0,44 & \multirow{6}{*}{0,789} \\
\hline 13 & Asisto puntualmente a las citas de control de diabetes & 0,30 & 0,65 & \\
\hline 14 & Asisto al laboratorio para realizar las pruebas respectivas & 0,31 & 0,65 & \\
\hline 15 & El equipo de salud me brinda información sobre mi enfermedad & 0,35 & 0,57 & \\
\hline 16 & Estoy satisfecho con los resultados del tratamiento médico & 0,32 & 0,52 & \\
\hline 17 & El tratamiento me permite controlar la enfermedad & 0,23 & 0,51 & \\
\hline 18 & Mi familia permite que prepare mis alimentos aparte del de los demás & 0,36 & 0,41 & \multirow{10}{*}{0,844} \\
\hline 19 & Mis familiares me facilitan los alimentos que necesito para la dieta especial & 0,46 & 0,59 & \\
\hline 20 & Mis familiares están pendientes de mis medicamentos durante el día & 0,49 & 0,66 & \\
\hline 21 & $\begin{array}{l}\text { Mis familiares colaboran con los implementos de uso personal especiales (zapatos, } \\
\text { limas para uñas) }\end{array}$ & 0,53 & 0,62 & \\
\hline 22 & $\begin{array}{l}\text { Recibo estímulos verbales (apoyo) de parte de mis familiares para que no abandone el } \\
\text { tratamiento }\end{array}$ & 0,38 & 0,58 & \\
\hline 23 & $\begin{array}{l}\text { Recibo premios o recompensas de parte de mis familiares para que no abandone el } \\
\text { tratamiento }\end{array}$ & 0,40 & 0,48 & \\
\hline 24 & Mis familiares me instan en la práctica de alguna actividad recreativa & 0,39 & 0,47 & \\
\hline 25 & Mis familiares me instan a la práctica de algún deporte & 0,37 & 0,45 & \\
\hline 26 & $\begin{array}{l}\text { Mis familiares están pendientes de que no pierda las citas de control de la diabetes } \\
\text { en el consultorio }\end{array}$ & 0,38 & 0,58 & \\
\hline 27 & $\begin{array}{l}\text { Mis familiares están pendientes de que el baño u otros lugares de la casa se } \\
\text { encuentren limpios y desinfectados }\end{array}$ & 0,36 & 0,60 & \\
\hline 28 & $\begin{array}{l}\text { Se realiza en la comunidad charlas educativas acerca de la diabetes con el fin de } \\
\text { informar a las personas }\end{array}$ & 0,33 & 0,58 & \multirow{6}{*}{0,787} \\
\hline 29 & En la comunidad donde vivo se organizan en grupos para la práctica de algún deporte & 0,30 & 0,53 & \\
\hline 30 & En la comunidad donde vivo se realizan actividades recreativas & 0,29 & 0,58 & \\
\hline 31 & $\begin{array}{l}\text { La comunidad se organiza para la realización de actividades como la compra de } \\
\text { medicamentos especiales para la diabetes }\end{array}$ & 0,31 & 0,58 & \\
\hline 32 & $\begin{array}{l}\text { La comunidad se organiza para la realización de actividades como la compra de } \\
\text { instrumentos de control de glucemia }\end{array}$ & 0,30 & 0,53 & \\
\hline 33 & Asisto a charlas brindadas por miembros del área de salud de mi comunidad & 0,39 & 0,53 & \\
\hline 34 & Mi familia conoce lo que es la diabetes mellitus & 0,40 & 0,44 & \multirow{3}{*}{0,717} \\
\hline 35 & Alguna vez he recibido información sobre la diabetes mellitus & 0,42 & 0,57 & \\
\hline 36 & Tengo claro en qué consiste la diabetes mellitus & 0,35 & 0,61 & \\
\hline & Total & & & 0,868 \\
\hline
\end{tabular}


Tabla 4. Eigenvalues y varianza del análisis de los componentes principales antes y después de la rotación vectorial

\begin{tabular}{|c|c|c|c|c|c|c|}
\hline \multirow[b]{2}{*}{$\mathbf{n}$} & \multicolumn{3}{|c|}{ Antes de la rotación vectorial } & \multicolumn{3}{|c|}{ Con rotación vectorial (Varimax) } \\
\hline & $\begin{array}{l}\text { Eigen- } \\
\text { value }\end{array}$ & $\begin{array}{c}\% \\
\text { Varianza }\end{array}$ & $\begin{array}{c}\% \text { Varianza } \\
\text { acumulativa }\end{array}$ & $\begin{array}{l}\text { Eigen- } \\
\text { value }\end{array}$ & $\begin{array}{c}\% \\
\text { Varianza }\end{array}$ & $\begin{array}{l}\% \text { Varianza } \\
\text { acumulativa }\end{array}$ \\
\hline Evito consumir golosinas o confites dulces & 6,531 & 18,142 & 18,142 & 4,455 & 12,375 & 12,375 \\
\hline Equilibro mi dieta consumiendo distintos tipos de alimentos & 4,151 & 11,531 & 29,673 & 3,805 & 10,570 & 22,945 \\
\hline Consumo frutas al menos una vez al día & 2,840 & 7,890 & 37,563 & 2,646 & 7,349 & 30,294 \\
\hline Camino para sentirme mejor & 2,670 & 7,416 & 44,980 & 2,471 & 6,864 & 37,158 \\
\hline Consumo alimentos antes de realizar ejercicio & 1,734 & 4,817 & 49,797 & 2,104 & 5,844 & 43,002 \\
\hline Tengo un horario fijo para realizar ejercicio & 1,529 & 4,247 & 54,044 & 1,997 & 5,546 & 48,548 \\
\hline $\begin{array}{l}\text { Sufro de menos cansancio cuando realizo alguna actividad } \\
\text { física }\end{array}$ & 1,385 & 3,848 & 57,892 & 1,854 & 5,150 & 53,698 \\
\hline $\begin{array}{l}\text { Me siento menos triste o decaído cuando realizo una } \\
\text { actividad física }\end{array}$ & 1,166 & 3,239 & 61,131 & 1,758 & 4,884 & 58,582 \\
\hline $\begin{array}{l}\text { Mejoro mi condición física cuando realizo alguna actividad } \\
\text { física }\end{array}$ & 1,116 & 3,100 & 64,231 & 1,748 & 4,856 & 63,438 \\
\hline $\begin{array}{l}\text { Siento mejores resultados en el tratamiento después } \\
\text { del ejercicio }\end{array}$ & 1,027 & 2,853 & 67,083 & 1,312 & 3,645 & 67,083 \\
\hline Realizo paseos cortos durante el día & 0,928 & 2,577 & 69,660 & & & \\
\hline $\begin{array}{l}\text { Visito al médico del consultorio en caso de alguna } \\
\text { complicación }\end{array}$ & 0,826 & 2,294 & 71,954 & & & \\
\hline Asisto puntualmente a las citas de control de diabetes & 0,768 & 2,133 & 74,087 & & & \\
\hline Asisto al laboratorio para realizar las pruebas respectivas & 0,728 & 2,022 & 76,108 & & & \\
\hline $\begin{array}{l}\text { El equipo de salud me brinda información sobre mi } \\
\text { enfermedad }\end{array}$ & 0,715 & 1,986 & 78,094 & & & \\
\hline Estoy satisfecho con los resultados del tratamiento médico & 0,659 & 1,830 & 79,924 & & & \\
\hline El tratamiento me permite controlar la enfermedad & 0,640 & 1,778 & 81,702 & & & \\
\hline $\begin{array}{l}\text { Mi familia permite que prepare mis alimentos aparte } \\
\text { del de los demás }\end{array}$ & 0,601 & 1,669 & 83,371 & & & \\
\hline $\begin{array}{l}\text { Mis familiares me facilitan los alimentos que necesito } \\
\text { para la dieta especial }\end{array}$ & 0,575 & 1,597 & 84,969 & & & \\
\hline $\begin{array}{l}\text { Mis familiares están pendientes de mis medicamentos } \\
\text { durante el día }\end{array}$ & 0,528 & 1,467 & 86,435 & & & \\
\hline $\begin{array}{l}\text { Mis familiares colaboran con los implementos de uso } \\
\text { personal especiales (zapatos, limas para uñas) }\end{array}$ & 0,503 & 1,398 & 87,833 & & & \\
\hline $\begin{array}{l}\text { Recibo estímulos verbales (apoyo) de parte de mis familiares } \\
\text { para que no abandone el tratamiento }\end{array}$ & 0,482 & 1,338 & 89,172 & & & \\
\hline $\begin{array}{l}\text { Recibo premios o recompensas de parte de mis familiares } \\
\text { para que no abandone el tratamiento }\end{array}$ & 0,427 & 1,185 & 90,357 & & & \\
\hline $\begin{array}{l}\text { Mis familiares me instan en la práctica de alguna actividad } \\
\text { recreativa }\end{array}$ & 0,408 & 1,134 & 91,491 & & & \\
\hline Mis familiares me instan a la práctica de algún deporte & 0,375 & 1,041 & 92,532 & & & \\
\hline $\begin{array}{l}\text { Mis familiares están pendientes de que no pierda las citas } \\
\text { de control de la diabetes en el Consultorio }\end{array}$ & 0,370 & 1,027 & 93,559 & & & \\
\hline
\end{tabular}




\section{Continuación Tabla 4.}

\begin{tabular}{|c|c|c|c|c|c|c|}
\hline \multirow[b]{2}{*}{$\mathbf{n}$} & \multicolumn{3}{|c|}{ Antes de la rotación vectorial } & \multirow{2}{*}{\multicolumn{3}{|c|}{$\begin{array}{l}\text { Con rotación vectorial (Varimax) } \\
\text { Eigen- } \% \quad \% \text { Varianza } \\
\text { value }\end{array}$}} \\
\hline & $\begin{array}{l}\text { Eigen- } \\
\text { value }\end{array}$ & $\begin{array}{c}\% \\
\text { Varianza }\end{array}$ & $\begin{array}{l}\% \text { Varianza } \\
\text { acumulativa }\end{array}$ & & & \\
\hline $\begin{array}{l}\text { Mis familiares están pendientes de que el baño u otros } \\
\text { lugares de la casa se encuentren limpios y desinfectados }\end{array}$ & 0,309 & 0,858 & 94,417 & & & \\
\hline $\begin{array}{l}\text { Se realiza en la comunidad charlas educativas acerca } \\
\text { de la diabetes con el fin de informar a las personas }\end{array}$ & 0,303 & 0,842 & 95,260 & & & \\
\hline $\begin{array}{l}\text { En la comunidad donde vivo se organizan en grupos } \\
\text { para la práctica de algún deporte }\end{array}$ & 0,291 & 0,808 & 96,068 & & & \\
\hline $\begin{array}{l}\text { En la comunidad donde vivo se realizan actividades } \\
\text { recreativas }\end{array}$ & 0,267 & 0,740 & 96,808 & & & \\
\hline $\begin{array}{l}\text { La comunidad se organiza para la realización de } \\
\text { actividades como la compra de medicamentos } \\
\text { especiales para la diabetes }\end{array}$ & 0,240 & 0,667 & 97,475 & & & \\
\hline $\begin{array}{l}\text { La comunidad se organiza para la realización de actividades } \\
\text { como la compra de instrumentos de control de glucemia }\end{array}$ & 0,232 & 0,646 & 98,121 & & & \\
\hline $\begin{array}{l}\text { Asisto a charlas brindadas por miembros del área de salud } \\
\text { de mi comunidad }\end{array}$ & 0,223 & 0,619 & 98,740 & & & \\
\hline Mi familia conoce lo que es la diabetes mellitus & 0,179 & 0,497 & 99,237 & & & \\
\hline Alguna vez he recibido información sobre la diabetes mellitus & 0,164 & 0,456 & 99,693 & & & \\
\hline Tengo claro en qué consiste la diabetes mellitus & 0,111 & 0,307 & 100,000 & & & \\
\hline
\end{tabular}

\section{Discusión}

A pesar de que el lenguaje utilizado en la versión original es igualmente el castellano, fue necesario hacer modificaciones a la escala a fin de facilitar su comprensión, especialmente en la utilización de vocablos específicos para Centroamérica en temas como la alimentación (ejemplo: manteca vegetal por margarina) o en los sistemas de atención (EBAIS por consultorio). Las entrevistas realizadas permitieron no sólo redactar los reactivos de manera tal que favoreciese su comprensión, sino además incorporar conductas que no contemplaba la versión original, pero que a juicio de los encuestados, se constituían en elementos que podían afectar su adherencia al tratamiento o a las prescripciones realizadas.

La escala adaptada en población chilena, obtenida a partir de la original, presentó una estructura factorial similar al modelo teórico original ${ }^{21}$, ya que la mayoría de los componentes se agrupan en las dimensiones correspondientes. Sin embargo, las dimensiones se redujeron a seis, debido a que la dimensión Valoración de la condición física se fusionó con la de ejercicio físico. Cabe destacar que se denominó a una dimensión Información, debido a que se agruparon nuevos ítems a los existentes en la dimensión original de higiene y autocuidado, pero que tenían como denominador común el acceso o manejo de información vinculada a la DM. El resto de las dimensiones provenientes del estudio original se mantuvieron. Adicionalmente, se eliminaron dos ítems con cargas cruzadas, por lo que la estructura factorial propuesta quedó conformada por 30 ítems y 6 factores. Pese a esto, el análisis confirmatorio no mostró un adecuado ajuste de los datos a esta solución, por lo que se hace necesario seguir profundizando el estudio de esta estructura, para lo que sería conveniente aumentar el n muestral.

Los factores sugeridos coinciden con los propuestos en diversos estudios en el campo de la adherencia en contextos geográficos y culturales distintos $^{22-33}$. Estos factores estarían relacionados al contexto sociocultural donde se expresan, dada la estrecha relación entre factores conductuales y culturales en la expresión de las actividades cotidianas como los horarios de comida, el tipo de alimento que el paciente consume, los hábitos establecidos en la persona y el tener o no conciencia de la propia enfermedad, en donde cobran especial relevancia las conductas de autocuidado y autoatención del paciente $^{18}$. 
Tabla 5. Medias y DE reactivos Escala EATDM III $\mathbf{R}$

\begin{tabular}{|c|c|c|c|c|c|c|}
\hline $\mathbf{n}$ & $\overline{\mathbf{x}}$ & DE & Mín. & Máx. & AS & CU \\
\hline Evito consumir golosinas o confites dulces & 2,97 & 1,281 & 0 & 4 & $-0,978$ & $-0,265$ \\
\hline Equilibro mi dieta consumiendo distintos tipos de alimentos & 3,27 & 1,079 & 0 & 4 & $-1,418$ & 1,104 \\
\hline Consumo frutas al menos una vez al día & 3,39 & 1,131 & 0 & 4 & $-1,708$ & 1,672 \\
\hline Camino para sentirme mejor & 2,46 & 1,519 & 0 & 4 & $-0,432$ & $-1,304$ \\
\hline Consumo alimentos antes de realizar ejercicio & 1,22 & 1,522 & 0 & 4 & 0,818 & $-0,923$ \\
\hline Tengo un horario fijo para realizar ejercicio & 1,10 & 1,500 & 0 & 4 & 1,031 & $-0,492$ \\
\hline Sufro de menos cansancio cuando realizo alguna actividad física & 1,74 & 1,617 & 0 & 4 & 0,221 & $-1,541$ \\
\hline Me siento menos triste o decaído cuando realizo una actividad física & 1,92 & 1,734 & 0 & 4 & 0,062 & $-1,741$ \\
\hline Mejoro mi condición física cuando realizo alguna actividad física & 2,25 & 1,756 & 0 & 4 & $-0,279$ & $-1,690$ \\
\hline Siento mejores resultados en el tratamiento después del ejercicio & 2,15 & 1,717 & 0 & 4 & $-0,195$ & $-1,674$ \\
\hline Realizo paseos cortos durante el día & 2,75 & 1,499 & 0 & 4 & $-0,759$ & $-0,944$ \\
\hline Visito al médico del consultorio en caso de alguna complicación & 3,31 & 1,315 & 0 & 4 & $-1,642$ & 1,134 \\
\hline Asisto puntualmente a las citas de control de diabetes & 3,61 & 1,012 & 0 & 4 & $-2,611$ & 5,643 \\
\hline Asisto al laboratorio para realizar las pruebas respectivas & 3,67 & 0,919 & 0 & 4 & $-2,825$ & 6,956 \\
\hline El equipo de salud me brinda información sobre mi enfermedad & 3,29 & 1,261 & 0 & 4 & $-1,650$ & 1,355 \\
\hline Estoy satisfecho con los resultados del tratamiento médico & 3,40 & 1,167 & 0 & 4 & $-1,918$ & 2,522 \\
\hline El tratamiento me permite controlar la enfermedad & 3,62 & 0,790 & 0 & 4 & $-2,219$ & 4,589 \\
\hline Mi familia permite que prepare mis alimentos aparte del de los demás & 2,70 & 1,623 & 0 & 4 & $-0,682$ & $-1,262$ \\
\hline Mis familiares me facilitan los alimentos que necesito para la dieta especial & 3,11 & 1,439 & 0 & 4 & $-1,343$ & 0,200 \\
\hline Mis familiares están pendientes de mis medicamentos durante el día & 2,82 & 1,648 & 0 & 4 & $-0,889$ & $-0,993$ \\
\hline $\begin{array}{l}\text { Mis familiares colaboran con los implementos de uso personal especiales } \\
\text { (zapatos, limas para uñas) }\end{array}$ & 2,55 & 1,762 & 0 & 4 & $-0,571$ & $-1,518$ \\
\hline $\begin{array}{l}\text { Recibo estímulos verbales (apoyo) de parte de mis familiares para que no } \\
\text { abandone el tratamiento }\end{array}$ & 3,11 & 1,504 & 0 & 4 & $-1,366$ & 0,140 \\
\hline $\begin{array}{l}\text { Recibo premios o recompensas de parte de mis familiares para que no } \\
\text { abandone el tratamiento }\end{array}$ & 1,31 & 1,660 & 0 & 4 & 0,750 & $-1,193$ \\
\hline Mis familiares me instan en la práctica de alguna actividad recreativa & 2,22 & 1,728 & 0 & 4 & $-0,202$ & $-1,705$ \\
\hline Mis familiares me instan a la práctica de algún deporte & 1,62 & 1,712 & 0 & 4 & 0,356 & $-1,629$ \\
\hline $\begin{array}{l}\text { Mis familiares están pendientes de que no pierda las citas de control de la } \\
\text { diabetes en el Consultorio }\end{array}$ & 2,84 & 1,668 & 0 & 4 & $-0,892$ & $-1,024$ \\
\hline $\begin{array}{l}\text { Mis familiares están pendientes de que el baño u otros lugares de la casa } \\
\text { se encuentren limpios y desinfectados }\end{array}$ & 3,37 & 1,320 & 0 & 4 & $-1,878$ & 1,906 \\
\hline $\begin{array}{l}\text { Se realiza en la comunidad charlas educativas acerca de la diabetes con } \\
\text { el fin de informar a las personas }\end{array}$ & 0,93 & 1,546 & 0 & 4 & 1,243 & $-0,233$ \\
\hline $\begin{array}{l}\text { En la comunidad donde vivo se organizan en grupos para la práctica } \\
\text { de algún deporte }\end{array}$ & 0,71 & 1,383 & 0 & 4 & 1,659 & 1,079 \\
\hline En la comunidad donde vivo se realizan actividades recreativas & 0,92 & 1,463 & 0 & 4 & 1,265 & $-0,005$ \\
\hline $\begin{array}{l}\text { La comunidad se organiza para la realización de actividades como la } \\
\text { compra de medicamentos especiales para la diabetes }\end{array}$ & 0,28 & 0,887 & 0 & 4 & 3,388 & 10,522 \\
\hline $\begin{array}{l}\text { La comunidad se organiza para la realización de actividades como la } \\
\text { compra de instrumentos de control de glucemia }\end{array}$ & 0,31 & 0,928 & 0 & 4 & 3,050 & 8,182 \\
\hline Asisto a charlas brindadas por miembros del área de salud de mi comunidad & 0,91 & 1,534 & 0 & 4 & 1,282 & $-0,137$ \\
\hline Mi familia conoce lo que es la diabetes mellitus & 3,15 & 1,384 & 0 & 4 & $-1,365$ & 0,349 \\
\hline Alguna vez he recibido información sobre la diabetes mellitus & 2,95 & 1,548 & 0 & 4 & $-1,102$ & $-0,466$ \\
\hline Tengo claro en qué consiste la diabetes mellitus & 3,13 & 1,357 & 0 & 4 & $-1,262$ & 0,156 \\
\hline
\end{tabular}


La consistencia interna del instrumento mostró una confiabilidad entre buena y excelente para la escala total $(0,87)$ y buena para todas las dimensiones, con excepción de Información $(0,72)$, y Dieta $(0,57)$. Esto tendría relación con la menor confiabilidad relacionada con la información que refieren los sujetos entrevistados sobre la DM y la declaración de evitar comer golosinas y favorecer comer variado y consumir frutas. Sin embargo, el alfa de Cronbach entrega sólo un estimado de la consistencia interna y de la estabilidad de la consistencia de la prueba, por lo que no se debiera utilizar bajo un criterio de decisión diagnóstico, y para su interpretación adecuada sería necesario realizar una estrategia de test-retest o de las formas equivalente, que no se pueden realizar con los datos utilizados para este trabajo. La consistencia interna hallada para la escala global es la adecuada para un instrumento de tamizaje, sin embargo, para su utilización como medida de uso clínico, se sugiere profundizar el estudio de los diversos ítems y dimensiones.

La dimensión Dieta, por ejemplo, consiste en 3 ítems que poseen una correlación entre 0,253 y 0,355 con un nivel de consistencia interna de 0,572 medido por alfa de Cronbach, lo que no supera los niveles sugeridos para su utilización en comparaciones individuales.

Una limitación al momento de la aplicación del EATDM-III, tiene relación con aspectos evolutivos, debido a que los adultos mayores de 65 años presentan dificultades como son la sordera, la retinopatía diabética o artritis. Otra limitación, se relaciona con las escasa información contenida en las fichas clínicas, observándose falta de datos completos y resultados de exámenes.

Es recomendable realizar otros estudios de verificación en distintas poblaciones, como usuarios de la atención secundaria de salud que ya estén siendo vistos por especialistas, o con diferentes grupos etarios como el grupo adolescente, a fin de mejorar su estructura factorial y apoyar los datos de validez del mismo. En necesario que los resultados pudieran validarse mediante un trazador biológico, como controles glicémicos periódicos de los pacientes encuestados, o con valores de hemoglobina glicosilada, como un indicador objetivo, toda vez que la normoglicemia en pacientes podría afirmar una adecuada adherencia al tratamiento.

Si bien el instrumento estudiado está en una versión preliminar, y se hace necesario replicar los resultados con un número mayor de participantes, el instrumento mostró indicadores aceptables de confiabilidad y validez, que permite su uso en pacientes diagnosticados con DMII (desde los 18 años en adelante), siendo un cuestionario genérico que mide factores vinculados a la adherencia terapéutica al tratamiento desde un punto de vista multidimensional y con un tiempo de administración reducido. Considerando lo anterior y las limitaciones antes expresadas, este instrumento podría ser utilizado para medir la adherencia al tratamiento en adultos que ya poseen el diagnóstico de DM2. Su aplicación podría ser positiva en los consultorios de atención primaria de salud, al ser el sector del sistema de salud que posee mayor cantidad de pacientes con DM2, permitiendo un control de la adherencia por el equipo de salud, lo que podría impactar en el tratamiento, pronóstico y calidad de vida de este grupo de pacientes.

\section{Referencias}

1. Encuesta Nacional de Salud. ENS Chile 2009-2010. Ministerio de Salud, Chile. Disponible en: http:// web.minsal.cl/portal/url/item/bcb03d7bc28b64dfe040010165012d23.pdf (consultado el 29 de agosto de 2014).

2. De Pablos P. La calidad de vida se resiente en los pacientes con diabetes. Revista Española de Economía de la Salud 2008; 7: 79-82.

3. Cárdenas V, Pedraza C, Lerma R. Calidad de vida del paciente con diabetes mellitus tipo 2. Ciencia UANL 2005; 8: 351-7.

4. Arcega A, Lara C, Ponce de León S. Factores relacionados con la percepción subjetiva de la calidad de vida de pacientes con diabetes. Rev Invest Clin 2005; 57: 676-84.

5. De los Ríos J, Sánchez J, Barrios P, Guerrero V. Calidad de vida en pacientes con diabetes mellitus tipo 2 . Rev Med Chile 2004; 42: 109-16.

6. Urzúa A. Calidad de vida relacionada con la salud: Aspectos conceptuales. Rev Med Chile 2010; 138: 341-8.

7. García A, Leiva F, Martos F, García A, Prados D, Sánchez de la Cuesta, et al. Calidad de vida en pacientes con hipertensión arterial y diabetes mellitus tipo 2. Medicina de familia 2001; 2: 29-34.

8. Vinaccia S, Fernández H, Quinceno J, López M, Otalvaro C. Calidad de vida relacionada con la salud y apoyo social funcional en pacientes diagnosticados con VIH/ SIDA. Ter Psicol 2008; 26: 125-32.

9. Mena F, Martín J, Simal F, Bellido J, Carretero J. Diabe- 
tes mellitus tipo 2 y calidad de vida relacionada con la salud: resultados del Estudio Hortega. An Med Interna 2006; 23: 357-60.

10. Hervas A, Zabaleta A, De Miguel G, Beldarrain O, Diez. Calidad de vida relacionada con la salud en pacientes con diabetes mellitus tipo 2. Anales Sis San Navarra 2007; 30: 45-52.

11. Urzúa A, Chirino A, Valladares G. Autoreporte de la calidad de vida relacionada con la salud en diabetes mellitus tipo 2. Rev Med Chile 2011; 139: 313-20.

12. Organización Mundial de la Salud. Adherencia a los Tratamientos a Largo Plazo: Pruebas Para La Acción 2004. Disponible en http://www.paho.org/Spanish/AD/ DPC/NC/nc-adherencia.htm [Consultado el 11 de julio de 2014].

13. Gigoux J, Moya P, Silva J. Adherencia al tratamiento farmacológico y relación con el control metabólico en pacientes con DM2. Rev Chil Pública; 2010; 14 (2-3): 238-9.

14. Ortiz M, Ortiz E. Psicología de la salud: una clave para conocer el fenómeno de la adherencia terapéutica. Rev Med Chile 2007; 135 (5): 647-52.

15. Rodríguez-Marín J. Efectos de la interacción entre el profesional sanitario y el paciente. Satisfacción del paciente. Cumplimiento de las prescripciones terapéuticas. En Rodríguez-Marín J, Ed. Psicología Social de la Salud. Madrid: Síntesis 1995; 151-60.

16. Cabrera A, del Castillo J, Domínguez S, Rodríguez M, Brito B, Borges C, et al. Estilo de vida y adherencia al tratamiento de la población canaria con diabetes mellitus tipo 2. Rev Esp Pública 2009; 83: 567-75.

17. Grau J, Martin L. La Investigación de la Adherencia Terapéutica como un Problema de la Psicología de la Salud. Psicología y Salud 2004; 14: 89-99.

18. Hoyos T, Arteaga M, Muñoz M. Factores de no adherencia al tratamiento en personas con diabetes mellitus tipo 2 en el domicilio. La visión del cuidador familiar. Invest Educ Enferm 2011; 29 (2): 194-203.

19. Ortiz M, Ortiz E, Gatica A, Gómez D. Factores Psicosociales Asociados a la Adherencia al Tratamiento de la diabetes mellitus tipo 2. Ter Psicol 2011; 29 (1): 5-11.

20. Villalobos A, Brenes J, Quiros D, León G. Características Psicométricas de la Escala de Adherencia al Tratamiento de la diabetes mellitus Tipo II-Versión III en una muestra de pacientes diabéticos de Costa Rica. Acta Colombiana de Psicología 2006; 9: 31-8.

21. Villalobos A, Araya C. Variables que afectan la adherencia al tratamiento en la diabetes mellitus tipo II y características poblacionales de una muestra de la zona norte de Costa Rica. Revista Costarricense de Psicología 2001; 33: 19-37.
22. Weinger K, Butler H, Welch G, La Greca A. Measuring Diabetes Self-Care: A psychometric analysis of Self-Care Inventory-revised with adults. Diabetes Care 2005; 28: 1346-52.

23. Klein S, Sheard N, Pi Sunyer X, Daly A, Wylie-roset J, Kulkarni K, et al. Weight management through lifestyle modification for the prevention and management of type 2 diabetes: rationale and strategies. A statement of the American Diabetes Association, the North American Association for the Study of Obesity, and the American Society for Clinical Nutrition. Am J Clin Nutr 2004; 80: 257-63.

24. Wenlk, Sheperd MD, Parchman ML. Family Support, Diet, and Exercise among Older Mexican Americans with Type 2 Diabetes. The Diabetes Educator 2004; 30 : 980-93.

25. Quiros D, Villalobos A. Comparación de factores vinculados a la adherencia al tratamiento en pacientes con diabetes mellitus tipo 2 en una muestra urbana y otra rural de Costa Rica. Univ Psychol 2007; 6: 679-88.

26. Balkrishnan R, Rajagolapan R, Camacho F, Huston S, Murray F, Anderson R. Predictors of medication adherence and associated health care costs in an older population with type 2 diabetes mellitus: A longitudinal cohort study. Clin Ther 2003; 25: 2958-71.

27. Harris S, Stewart M, Brown J, Wetmores S, Faulds C, Webster-Bogaert $S$, et al. Type 2 diabetes in family practice. Room for improvement. Can Fam Physician 2003; 49: 778-85.

28. Fisher E, Walker E, Bostrom A, Fischhoff B, Haire-joshu $\mathrm{D}$, Bennette-Johnson S. Behavioral Science Research in the Prevention of Diabetes. Diabetes Care 2002; 25: 599606.

29. Nelson K, Reiber G, Boyko E. Diet and Exercise Among Adults With Type 2 Diabetes. Diabetes Care 2002; 25: 1722-8.

30. Ciechanowski P, Katon W, Russo J, Walker E. The Patient-Provider Relationship: Attachment Theory and Adherence to Treatment in Diabetes. Am J Psychiatry 2001; 158: 29-35.

31. Hamdy O, Horton E. Diet and Exercise in type 2 diabetes mellitus. Endocrinol Metab Clin North Am 2001; 30: 883-907.

32. Norris S, Engelgau M, Venkat K. Effectiveness of Self-Management Training in Type 2 Diabetes. A systematic review of randomized controlled trials. Diabetes Care 2001; 24: 561-87.

33. Scientific Advisory Committee Of The Medical Outcomes Trust. Assessing health status and quality of life instruments: attribute and review criteria. Qual life Res 2002; 11: 193-205. 